\title{
THE QUASI-ROCHE LOBE OVERFLOW STATE IN THE EVOLUTION OF CLOSE BINARY SYSTEMS CONTAINING A RADIO PULSAR
}

\author{
O. G. Benvenuto ${ }^{1,3}$, M. A. De Vito ${ }^{1,4}$, and J. E. Horvath ${ }^{2}$ \\ ${ }^{1}$ Facultad de Ciencias Astronómicas y Geofísicas, Universidad Nacional de La Plata and Instituto de Astrofísica de La Plata (IALP), \\ CCT-CONICET-UNLP. Paseo del Bosque S/N (B1900FWA), La Plata, Argentina; adevito@ fcaglp.unlp.edu.ar \\ 2 Instituto de Astronomia, Geofísica e Ciências Atmosféricas, Universidade de São Paulo R. do Matão 1226 (05508-090), \\ Cidade Universitária, São Paulo SP, Brazil; foton@iag.usp.br \\ Received 2014 June 30; accepted 2014 October 23; published 2014 December 18
}

\begin{abstract}
We study the evolution of close binary systems formed by a normal (solar composition), intermediate-mass-donor star together with a neutron star. We consider models including irradiation feedback and evaporation. These nonstandard ingredients deeply modify the mass-transfer stages of these binaries. While models that neglect irradiation feedback undergo continuous, long-standing mass-transfer episodes, models including these effects suffer a number of cycles of mass transfer and detachment. During mass transfer, the systems should reveal themselves as low-mass X-ray binaries (LMXBs), whereas when they are detached they behave as binary radio pulsars. We show that at these stages irradiated models are in a Roche lobe overflow (RLOF) state or in a quasiRLOF state. Quasi-RLOF stars have radii slightly smaller than their Roche lobes. Remarkably, these conditions are attained for an orbital period as well as donor mass values in the range corresponding to a family of binary radio pulsars known as "redbacks." Thus, redback companions should be quasi-RLOF stars. We show that the characteristics of the redback system PSR J1723-2837 are accounted for by these models. In each mass-transfer cycle these systems should switch from LMXB to binary radio pulsar states with a timescale of approximately one million years. However, there is recent and fast growing evidence of systems switching on far shorter, human timescales. This should be related to instabilities in the accretion disk surrounding the neutron star and/or radio ejection, still to be included in the model having the quasi-RLOF state as a general condition.
\end{abstract}

Key words: binaries: close - pulsars: general - pulsars: individual (PSR J1723-2837) - stars: evolution

\section{INTRODUCTION}

Today, it is usually accepted that millisecond pulsars (MSPs) are old neutron stars (NSs), recycled in close binary systems (CBSs; Alpar et al. 1982). In this process, the companion (a low-mass star) transfers mass and angular momentum to the NS during a Roche Lobe OverFlow (RLOF) episode, increasing the mass and rotation rate of the NS. During the mass-transfer process, the system is a copious source of X-rays, generally (but not only) detectable as a low-mass X-ray binary (LMXB). For these types of systems, the standard models of CBSs predict a long and stable episode of mass transfer, as a consequence of the evolution of the donor star, with a small number of RLOFs due to thermonuclear flashes (see, e.g., Webbink et al. 1983; Tauris \& Savonije 1999; Podsiadlowski, Rappaport, \& Pfahl 2002; Benvenuto \& De Vito 2005). As a final result, the formation of an MSP (with a spin period of $P_{\text {rot }}<10 \mathrm{~ms}$ ) is expected. The most common companion is a low-mass helium white dwarf (HeWD), with characteristic mass values of $M_{2} \sim 0.2-0.3 M_{\odot} .^{5}$ These objects are compact remnants of CBSs with $P_{\text {orb }}>1$ day (where $P_{\text {orb }}$ is the orbital period of the system). Other physical phenomena that play relevant roles in the formation of MSPs are magnetic braking and gravitational radiation; see, e.g., Verbunt (1993) for a review.

Standard models support the idea that MSPs descend from LMXBs; however, the existence of many isolated MSPs represents a serious difficulty faced by the model. Nevertheless,

\footnotetext{
3 Member of the Carrera del Investigador Científico of the Comisión de Investigaciones Científicas (CIC) de la Provincia de Buenos Aires, Argentina.

4 Member of the Carrera del Investigador Científico, CONICET, Argentina.

5 As usual, we denote the quantities related to the NS and donor/companion star with subscripts NS and 2, respectively.
}

Fruchter et al. (1988) discovered an MSP in an eclipsing binary system (PSR 1957+20). It has a spin period of $1.6 \mathrm{~ms}$, in orbit around a low-mass companion of only $\sim 0.02 M_{\odot}$. Due to the strong eclipses and the small mass of the companion, it was proposed that pulsar radiation is ablating the companion (Phinney et al. 1988). This is the representative object of a class of eclipsing binary systems, composed by an MSP and a very-low-mass companion $\left(M_{2}<0.05 M_{\odot}\right)$, in tight orbits $\left(P_{\text {orb }}<1\right.$ day $)$, generally called "black widows." The evaporation of the companion by the pulsar emission in CBSs could be a process that allows recycled NSs to unbind their companions and finally appear as isolated MSPs.

It has recently been shown (Benvenuto et al. 2014, hereafter $\mathrm{BDVH})$ that evolutionary trajectories leading to the evaporating (black widows) locus to cross a region in the $P_{\text {orb }}-M_{2}$ plane in which accretion-driven irradiation causes an evolution markedly different from the standard one leading to LMXBs. In fact, there is a wide zone populated by pulsar companions that standard models of close binary evolution cannot reproduce. It is precisely this stage that will be studied in this paper.

Some time ago it was proposed that the pressure due to radio pulsar irradiation may preclude accretion onto the NS. This phenomenon is usually referred to as "radio ejection" (Ruderman et al. 1989; Burderi et al. 2001). If radio ejection were fully effective in inhibiting accretion, the results described in BDVH would no longer be valid. Also, evaporation may be relevant at stages earlier than those corresponding to black widows (Chen et al. 2013).

Perhaps the most important nonstandard effect in the early stages of CBSs is irradiation feedback (Büning \& Ritter 2004). The accretion onto the NS releases X-rays, which are partially absorbed by the companion and produce a partial blockage 
of the energy that emerges from the donor star interior. As a nontrivial result, the mass-transfer episode becomes cyclic (see Büning \& Ritter 2004; Benvenuto et al. 2012). Calculations (see BDVH) predict a back-and-forth switch of the system between LMXB and binary radio pulsar states. Irradiation swells the companion and the latter is filling, or quasi-filling its Roche lobe. The inclusion of this new ingredient allowed us to perform more realistic calculations, and to reconcile the theory with observations.

Direct proof of the theoretical scenario for recycling old NSs in MSPs was found for the first time in SAX 1808.4-3658. Wijnands \& van der Klis (1998) reported coherent millisecond $\mathrm{X}$-ray pulsations in the persistent flux of this CBS. The source will probably become an MSP when accretion turns off. The parameters $\left(P_{\mathrm{orb}}=2 \mathrm{hr}\right.$, eccentricity $e<5 \times 10^{-4}$ and pulsar mass function $f_{\mathrm{NS}}=3.7789(2) \times 10^{-5} M_{\odot}$; Chakrabarty \& Morgan 1998) imply a companion mass of $M_{2}=0.14-0.18 M_{\odot}$, if the NS mass is in the range $M_{\mathrm{NS}}=1.35-2.0 M_{\odot}$. The orbital period $\left(P_{\text {orb }}<1\right.$ day) and the mass of the companion $\left(0.1<M_{2} / M_{\odot}<0.4\right)$ place this system in the redback region (Roberts 2013a) in the plane $P_{\text {orb }}-M_{2}$. Burderi \& Di Salvo (2013) have proposed that this object is undergoing a switch between accretion and MSP stages dominated by radio ejection.

A handful of systems placed in the redback region have shown clear signs of disk states and extended radius of the donor (although not always simultaneously). For example, the FIRST J102347.67+003841.2 source with $P_{\text {orb }}=4.75 \mathrm{hr}$ (Thorstensen \& Armstrong 2005) is coincident with the MSP PSR J1023+0038 (Archibald et al. 2009). The 2001 observations showed evidence of mass outflow, but since 2002 a quiescent state followed. Later, it was found that for reasonable NS masses, the companion mass is limited to $0.14 \lesssim M_{2} / M_{\odot} \lesssim 0.42$ and likely fills its Roche lobe because of the presence of ionized material. The recent (mid-2013) enhancement of UV and X-ray emission (Stappers et al. 2013; Patruno et al. 2014), together with the interruption of the radio pulsations, has been interpreted as evidence that the LMXB phase returned (Archibald et al. 2009). Since the system is far from any globular cluster and the orbit is almost circular, it is reasonable to assume that the pulsar has been recycled by the companion and alternates between two states with the companion filling the Roche lobe. This system may be seen as a redback in the MSP phase and turn to LMXB phenomenology when accretion (re)starts.

Redbacks seemed to be rare, but since 2009 many of them have been discovered in the Galactic plane (Roberts 2013a), as well as in globular clusters (see Pulsars in Globular Clusters, Freire's homepage ${ }^{6}$ ). Currently, there are $\sim 7$ redbacks known in the Galactic field, and $\sim 12$ in globular clusters.

Perhaps the best example of this class of objects, changing its state between accreting X-ray MSP and radio pulsars state is precisely a system located in the globular cluster M28, PSR J1824-2452I (with $P_{\text {rot }}=3.9 \mathrm{~ms}$ and $P_{\text {orb }}=11 \mathrm{hr}$ ). The system was detected switching from an accretion phase to a radio pulsar phase in the last decade (Papitto et al. 2013). The change between rotation and accretion powered states in a few days to few months mark the existence of a phenomenon that occurs on a timescale much shorter that the usual long scale of the order of $\sim$ gigayears in these type of CBSs, or the pulsed mass transfer scale recently found in BDVH.

Further systems belonging to the redback family and displaying evidence of an extended radius of the companion, even

\footnotetext{
6 http://www.naic.edu/ pfreire/GCpsr.html
}

in quiescence, include PSR J1723-2837. This is an eclipsing, $P_{\text {rot }}=1.86 \mathrm{~ms}$ binary radio pulsar in an almost circular orbit with $P_{\text {orb }}=14.76 \mathrm{hr}$ discovered by Faulkner et al. (2004). The eclipses suggested that the pulsar companion is a nondegenerate, extended star. This has been confirmed by Crawford et al. (2013), who identify the companion in the IR, optical, and UV bands. The spectral analysis put the companion between $\mathrm{G} 5$ and $\mathrm{K} 0$ spectral types with an effective temperature of $5000-6000 \mathrm{~K}$ and a surface gravity reasonably consistent with a main-sequence star. These authors derived a mass ratio of $M_{\mathrm{NS}} / M_{2}=3.3 \pm 0.5$, giving a companion mass range of $M_{2}=0.4-0.7 M_{\odot}$, and an orbital inclination angle between $30^{\circ}-41^{\circ}$, assuming a pulsar mass in the range of $M_{\mathrm{NS}}=1.4-2.0 M_{\odot}$. The derived radius of the companion is larger than expected, likely close to filling its Roche lobe, i.e., a quasi-RLOF object.

An example of a quasi-RLOF object is PSR J1740-5340. It is a binary MSP ( $P_{\text {rot }}=3.65 \mathrm{~ms}$ ) that has $P_{\text {orb }}=32.5 \mathrm{hr}$, which falls outside the usually considered range of values corresponding to redbacks. For this object Burderi et al. (2002) proposed a possible binary progenitor that accounts for the observational data of the companion and considers that it is on a radio ejection stage.

XSS J12270-4859, on the other hand, experienced a transition from an LMXB state to a radio MSP (Bassa et al. 2014; Roy et al. 2014). This report confirm the idea that this pair belongs to the group formed by a low-mass quasi-RLOF object and a rotation-powered MSP, a redback system in the current state of XSS J12270-4859.

Finally, we quote the cases of PSR J1023+0038 and XSS J12270-4859, which experienced transitions in opposite directions. In the transition of PSR J1023+0038 from MSP to LMXB, an increase in the flux of $\gamma$-rays was detected (Stappers et al. 2013). XSS J12270-4859 showed a decrease of the $\gamma$-ray brightness (Tam et al 2013), though less intense than the growth in the case of PSR J1023+0038. The transition of PSR J1023+0038 occurs in $\sim 2$ weeks, while the transition of XSS J12270-4859 was in $\sim 5$ weeks. The process of back-andforth transitions seem to be fast.

Besides the eclipses and a nondegenerate low-mass companion, redbacks seem to have another feature that identified them: the high filling factor of the Roche lobe by the donor star component ( $~ 290 \%$ in many cases, see Roberts $2013 \mathrm{~b}$ and references therein).

All the phenomenology described above cannot be solely related to the evolution of the donor star, because the latter has a typical timescale that is far greater. It seems more natural to interpret the changes between LMXB and the binary radio pulsar as due to instabilities of the accretion disk surrounding the NS. However, for these instabilities to occur, the companion star must act as a donor. If we observe a binary MSP showing redback features, it would be hard to distinguish whether it was visible because of disk instabilities or if it was detached due to donor evolution. A population synthesis should be helpful to discern between these possible situations, because the time fraction of the cycle in which mass transfer occurs is small (see $\mathrm{BDVH}$ ) and this is the only situation in which the pulsar should be detectable due to accretion disk instabilities (otherwise, there is no accretion). If the scenario we are suggesting here is at least qualitatively correct, we should expect that redbacks should be more abundant than LMXBs with orbital periods and companion masses in the same range. Unfortunately, it seems that the number of already detected systems of these kinds prevent us 
from performing a solid statistical inference. In any case, the very existence of quasi-RLOF stars should be related to the irradiation feedback phenomenon.

It is the aim of this paper to discuss the quasi-RLOF state and also to show that the evolutionary models presented in BDVH provide a natural account of the characteristics of the redback system PSR J1723-2837.

The remainder of this paper is organized as follows. In Section 2, we briefly describe the ingredients of our stellar code. In Section 3, we present the quasi-RLOF state in the evolution of CBSs containing radio pulsars. In Section 4 , we apply this novel evolutionary state to the case of the available observations of the PSR J1723-2837 redback system. In Section 5, we discuss the meaning of the results presented in this paper and, finally, in Section 6, we give some concluding remarks.

\section{THE NUMERICAL CODE}

The results to be described below have been found employing our CBS evolutionary code (Benvenuto \& De Vito 2003) updated by the inclusion of irradiation feedback and evaporation. During a RLOF episode, the code solves for the donor star structure, the instantaneous mass-transfer rate $\dot{M}$, both masses, and the orbital semi axis implicitly. This method allows for the computation of the mass-transfer cycles quoted in Section 1 to be presented below in detail. In detached conditions, the code employs the standard Henyey technique.

We assumed that the NS accretes a fraction $\beta$ of the transferred matter up to the Eddington critical rate $\dot{M}_{\text {Edd }} \approx 2 \times 10^{-8} M_{\odot} \mathrm{y}^{-1}$, while very-low-accretion rates $\left(\lesssim 1.3 \times 10^{-11} M_{\odot} \mathrm{y}^{-1}\right)$ are inhibited by the propeller mechanism (Romanova et al. 2008). Angular momentum sinks due to gravitational radiation, magnetic braking, and mass loss from the system have been treated as in Benvenuto \& De Vito (2003).

Irradiation feedback has been included following Hameury \& Ritter (1997) who replace the usual relation between luminosity, radius, and effective temperature $L=4 \pi R_{2}^{2} \sigma T_{\text {eff }}^{4}$ by

$$
L=R_{2}^{2} \sigma T_{\mathrm{eff}, 0}^{4} \int_{0}^{2 \pi} \int_{0}^{\pi} G(x(\theta, \phi)) \sin \theta d \theta d \phi,
$$

where $\sigma$ is the Stefan-Boltzmann constant, $T_{\mathrm{eff}, 0}$ is the effective temperature of the nonilluminated part of the star, $G(x)=$ $\left(T_{\text {eff }}(x) / T_{\text {eff }, 0}\right)^{4}-x$ and $x=F_{\text {irrad }} /\left(\sigma T_{\text {eff }, 0}^{4}\right)$, where $F_{\text {irrad }}$ is the incident irradiating flux. $T_{\text {eff }}(x)$ is computed assuming that at deep enough layers, perturbations due to irradiation must vanish (Vaz \& Nordlund 1985).

We assume that the NS acts as an isotropic point source, releasing an accretion luminosity $L_{\mathrm{acc}}=G M_{\mathrm{NS}} \dot{M}_{\mathrm{NS}} / R_{\mathrm{NS}}(G$ is the gravitational constant and $R_{\mathrm{NS}}$, and $\dot{M}_{\mathrm{NS}}$ are the radius and accretion rate of the NS, respectively). The irradiation flux incident onto the donor star, effectively participating in the feedback phenomenon is $F_{\text {irrad }}=\alpha_{\text {irrad }} L_{\text {acc }} /\left(4 \pi a^{2}\right)$, where $\alpha_{\text {irrad }}$ is the fraction of the flux irradiated toward the donor that drives the phenomenon, and $a$ is the orbital radius. Because of the assumption of isotropy and the way it has been defined, we have $0 \leqslant \alpha_{\text {irrad }} \leqslant 1$.

Because we assume in the models presented below that evaporation does not play a role, we shall not detail the way we handled this phenomenon in our code. For further details, we refer the reader to BDVH and references therein.

\section{THE QUASI-ROCHE LOBE OVERFLOW STATE}

The occurrence of the quasi-RLOF state is easy to understand by performing a close examination of the evolution of the donor mass when irradiation feedback is considered. Before the onset of a RLOF, we assume there is no accretion onto the NS; thus, there is no irradiation incident onto the donor star. Just after the beginning of the RLOF, irradiation feedback forces the donor star to have an instantaneous mass-transfer rate $\dot{M}$ that overflows the value it would have had if irradiation were neglected. Let us define $\Delta \dot{M} \equiv \max \left(\dot{M}-\dot{M}_{\text {nif }}\right)$ the maximum mass-loss rate of irradiated models minus the $\dot{M}_{\text {nif }}$ value it would have had without irradiation feedback. The general trend is that the stronger the irradiation regime, the larger $\Delta \dot{M}$.

In order to understand the quasi-RLOF state, for the moment, let us assume that the donor star fulfills the conditions in which undergoes cyclic mass transfer for all values of $\alpha_{\text {irrad }}>0$.

Initially, irradiation feedback leads to $\Delta \dot{M}>0$. After a time of the order of the Kelvin-Helmholtz timescale $\tau_{\mathrm{KH}}$ (where $\tau_{\mathrm{KH}}=G M_{2}^{2} /\left(R_{2} L_{2}\right)$, and $R_{2}$ and $L_{2}$ are the radius and luminosity of the donor star, respectively) the star relaxes, damping the perturbation due to irradiation. Then, the donor star contracts because it has a mass lower than it would need to stay in a semi-detached state. Thus, the RLOF ends. While the stellar envelope reacts this way, the deep interior has a chemical profile evolving due to nuclear reactions and mixing. Very often, this causes the star to swell enough, leading to the occurrence of another RLOF on a nuclear timescale. This is the very reason why stars may undergo a cyclic behavior. The time the star needs to spend to reach other RLOF conditions (if any) depends on the value of $\Delta \dot{M}$. If irradiation feedback is weak (e.g., the case of $\left.\alpha_{\text {irrad }}=0.01\right) \Delta \dot{M}$ is small and the star needs to evolve its internal chemical profile very little to RLOF again. Therefore, the average duration of mass transfer cycles is rather short, leading to the occurrence of a large number of cycles. On the contrary, if irradiation feedback is stronger (e.g., up to $\left.\alpha_{\text {irrad }}=1.00\right), \Delta \dot{M}$ will be larger, the star will need more time to evolve and swell enough to RLOF, again mass-transfer cycles will be longer and the star will undergo a much lower number of cycles.

The qualitative description given above can be analyzed with the help of Figure 1. There, we compare the evolution of the donor star for systems that initially had a normal star of $1.50 M_{\odot}$, a "canonical" NS of $1.4 M_{\odot}$, and a one-day orbital period. We kept fixed $\beta=0.5$ and, in order to explore the whole parameter space of irradiation, we considered values of $\alpha_{\text {irrad }}=0.00,0.01,0.10$, and 1.00. Here we have modeled the long timescale evolution of the systems.

In the upper and middle panels of Figure 1, we display the mass-transfer rate and the mass for these systems, complying with the behavior described above. While the model without irradiation shows a smooth evolution, irradiated models follow a step-like behavior in the mass. These steps are smaller the weaker the irradiation strength.

In the lower panel of Figure 1, we show the evolution of the radius of the equivalent Roche lobe against the stellar radius for the case of $\alpha_{\text {irrad }}=0.10$. It is apparent that these radii are almost the same during RLOFs (the difference of these radii is very small, being of the order of the pressure scale height $H_{p}=d r / d \log P$ at the photosphere), while after detachment the star becomes slightly smaller than its associated Roche lobe. The star detaches because it has lost more mass than the amount it would have if irradiation feedback were absent. In the latter conditions, the star would be on a RLOF continuously. This represents a rather small perturbation to the stellar structure. Thus, there is no possibility for the donor star other than to have a radius slightly smaller than its Roche lobe. The donor star must settle on a quasi-RLOF state. 


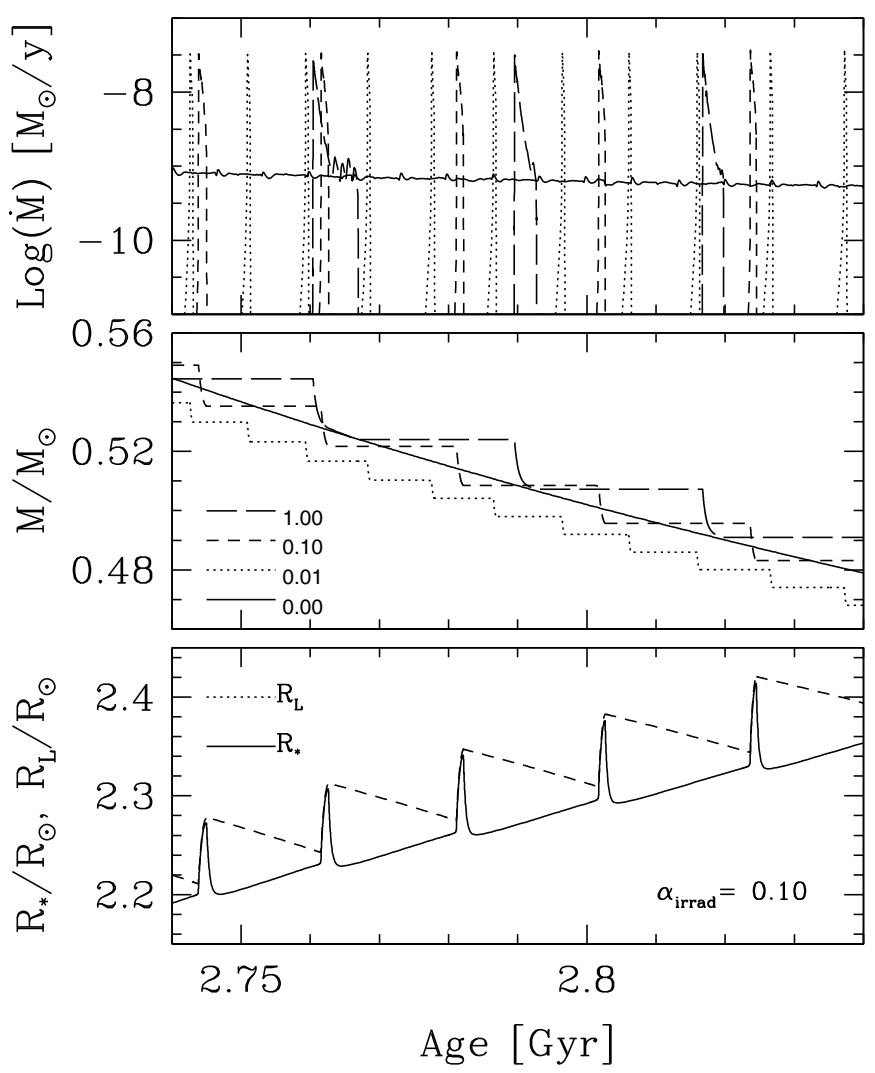

Figure 1. Evolution of a donor star that initially has a mass of $1.50 \mathrm{M}_{\odot}$, together with an NS of $1.4 M_{\odot},(\beta=0.5)$ on a one-day orbital period, neglecting irradiation and also considering it with $\alpha_{\text {irrad }}=0.01,0.10$, and 1.00 . The upper panel shows the mass-transfer cycles while the middle panel shows the corresponding evolution of the donor mass. The lower panel shows the evolution of the radius of the donor star and the equivalent Roche lobe. There, for the sake of clarity, we only considered the case of $\alpha_{\text {irrad }}=0.10$. It is noticeable that after detachments, the star is in the quasi-RLOF state.

These arguments are quite general in the sense that if an irradiated donor star undergoes cyclic mass transfer, it must be in the RLOF or in the quasi-RLOF state. This provides a natural explanation for the occurrence of redback pulsars. Redbacks are a rapidly growing family of eclipsing binary systems containing an MSP together with a donor star with a mass of $\sim 0.10 M_{\odot}$ and orbital periods from 0.1 to 1.0 days. As described in BDVH, some redbacks evolve to the black widow state, while others give rise to the formation of low-mass, HeWD-pulsar system. Our models support the idea that all redbacks must have a nondegenerate companion in the quasi-RLOF state. This may also be the case for more massive objects usually interpreted as carbon-oxygen WD companions to pulsars (see Figure 3 of $\mathrm{BDVH}$ ).

The very existence of the quasi-RLOF state indicates that pulsars may be observed accompanied not only by WDs but also by nondegenerate companions (in fact, the deep interior of the donor companion should be at densities high enough for the electrons to become semi-degenerate, but this is not the case of the extended envelope). It also proves the relevance of considering irradiation feedback in CBS evolution.

\section{APPLICATION TO THE CASE OF THE PSR J1723-2837 REDBACK SYSTEM}

As stated above, the system containing PSR J1723-2837 is one of the best observed cases to be explained as a prototype. In order to interpret the available observations of PSR J1723-2837 in the framework of an evolutionary model, we have searched among the set of calculations presented in BDVH. We found that the CBSs formed by a normal, solar composition $1.25 M_{\odot}$ star together with an NS of $1.40 M_{\odot}$ on a 0.75 day circular orbit are suitable for such purposes. We shall consider models with and without irradiation feedback. With the purpose of exploring the whole parameter space of irradiation, we considered four values of the coupling constant $\alpha_{\text {irrad }}=0.00,0.01,0.10$, and 1.00. The comparison of the available data on PSR J1723-2837 and the evolutionary tracks will be performed with the help of Figures 2-7

In Figure 2, we present the evolutionary tracks in the HR diagram for each value of $\alpha_{\text {irrad}}$, where we indicate the range of effective temperatures compatible with the spectroscopic observations corresponding to the donor star presented by Crawford et al. (2013). There are two epochs compatible with observations, one before bending blueward and another after it. In both cases, the star has surface conditions similar to those of main-sequence stars, but its internal structure is quite different.

Figure 3 depicts the donor mass versus orbital period relationship for the considered systems. The nonirradiated model reaches the observed orbital period of PSR J1723-2837 in three epochs while irradiated models fulfill this condition several times. In all cases, the mass range at which the correct period is reached corresponds to the donor mass range given by Crawford et al. (2013). The evolution of the surface gravity is shown in Figure 4. Again, these values are similar to those of main-sequence stars, as can be expected by the results included in Figure 2. The mass-transfer rate for these systems is given in Figure 5. Models considering irradiation feedback are consistent with the observed phenomenology, since we do not observe an LMXB at all times.

Figure 6 denotes the mass ratio of the components of the systems considered above. The observed value of $M_{\mathrm{NS}} / M_{2}=$ $3.3 \pm 0.5$ is attained at an age of $\sim 4.9$ Gyr. Finally, in Figure 7 , we present the unfilled fraction of the Roche lobe as a function of time. Irradiated, detached models have radii smaller than the corresponding Roche lobe in, at most, a few percent. Clearly, the donor star is in the quasi-RLOF state as indicated by observations.

In the frame of our models, a CBS capable to account for the properties of the PSR J1723-2837 system has to undergo cyclic mass-transfer episodes. If, on the contrary, mass transfer were continuous, we would observe the system as an LMXB. Our models provide the observed mass ratio of the PSR J1723-2837 system for an age of $\sim 4.9 \mathrm{Gyr}$, which corresponds to the portion of the evolutionary track presented in Figure 2 before bending blueward. At this age, the models with $\alpha_{\text {irrad }}=0.00,0.10$, and 1.00 do not undergo cyclic mass transfer, meanwhile that with $\alpha_{\text {irrad }}=0.01$ does. Thus, low-irradiation regimes provide a satisfactory account of the properties observed of the donor star in PSR J1723-2837 system.

\section{DISCUSSION}

In the previous section, we have discussed the relevance of evolutionary tracks including irradiation feedback to the case of the redback system PSR J1723-2837. We found that the observations reported by Crawford et al. (2013) are consistent with the theoretical picture including irradiation. Moreover, the analysis presented in Section 4 indicates that the quasiRLOF state is reached by actual binary systems. In view of the generality of the arguments presented above, we suggest that 


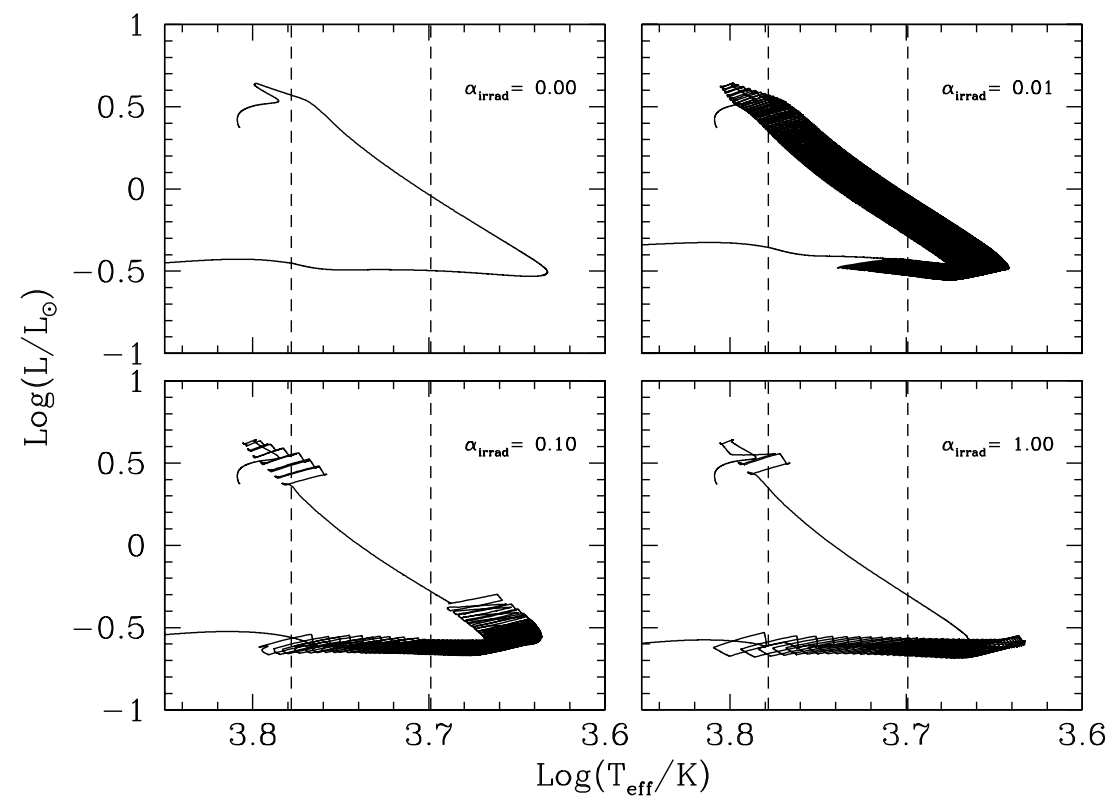

Figure 2. Evolutionary tracks for the donor star of a system formed by a normal, solar composition donor star of $1.25 M_{\odot}$, evolving on a CBS together with a $1.4 M_{\odot}$ mass NS on an 0.75 day orbit. Each panel corresponds to different irradiation feedback regimes indicated with the corresponding value of $\alpha_{\text {irrad }}$. The horizontal axis corresponds to the effective temperature of the non-irradiated portion of the stellar surface. Vertical dashed lines indicate the range of effective temperatures for the donor star in the PSR J1723-2837 system (Crawford et al. 2013).

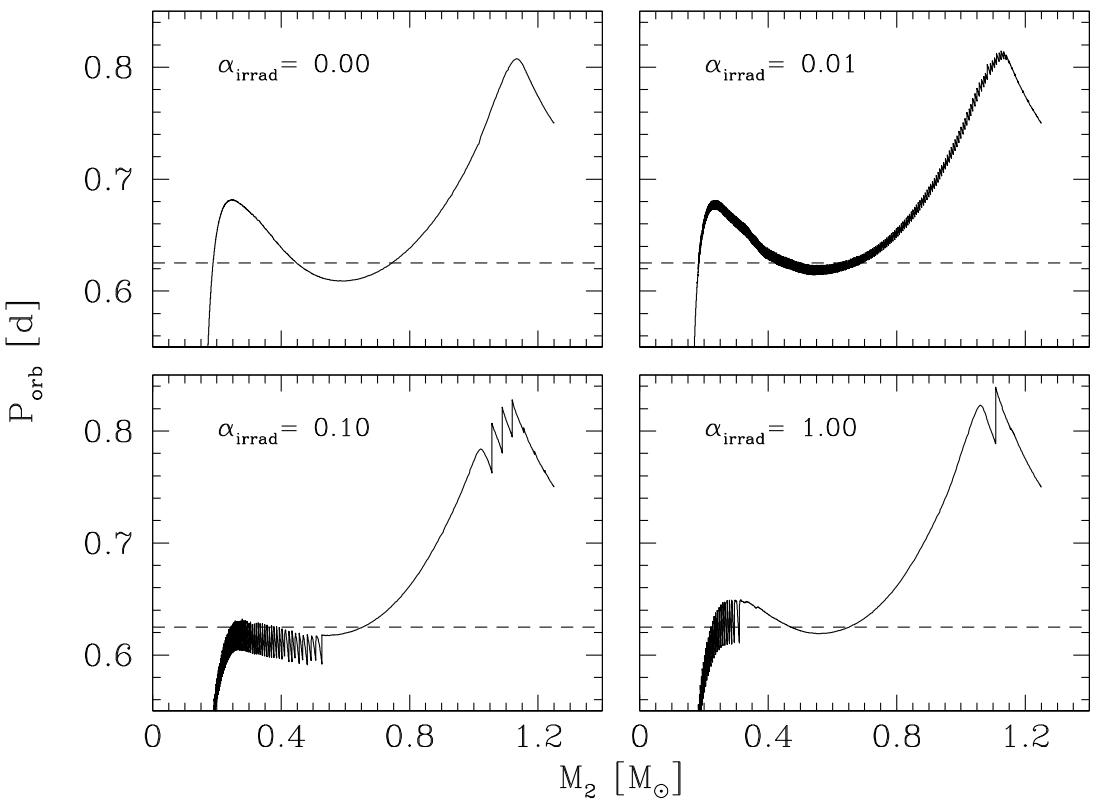

Figure 3. Donor mass vs. orbital period for the systems included in Figure 2. The orbital period corresponding to the PSR J1723-2837 system is denoted with a horizontal dashed line. Notice that irradiated models reach the observed period value several times while the nonirradiated one provides three values for the donor mass.

essentially all redbacks should have quasi-RLOF companions. In other words, that redbacks are the observational manifestation of the quasi-RLOF state. This is supported by the results presented in Roberts (2013b), particularly the data related to the Roche lobe filling factors.

In this study, we have considered a single value for the parameter $\beta(=0.5)$. This quantity is the fraction of the matter the NS is assumed to accrete $\left(\dot{M}_{\mathrm{NS}}=\min \left[-\beta \dot{M}_{2}, \dot{M}_{\text {Edd }}\right]\right)$. While it is well-known that the evolution of donor stars is largely independent of $\beta$, the mass of the NS is sensitively affected by this choice. There have been some studies indicating that the value of $\beta$ may be lower in some cases (see, e.g., Benvenuto et al. 2006 for the case of PSR J1713+0747). If the NS mass growth were slower, this would modify the results presented in Figure 6; and a good fit to the PSR J1723-2837 system would be provided by different initial conditions. In any case, we should remark that the initial NS mass value is, to a large extent, also uncertain. Evidently, there is some degeneracy for the initial parameters of these binary systems, and thus we are not claiming that the evolutionary tracks we considered here in Section 4 are the only possible ones. However, while finding the results presented in this paper, we did not perform any fine tuning of the evolutionary model's parameters, and yet we found very suitable calculations for a previously computed set. 


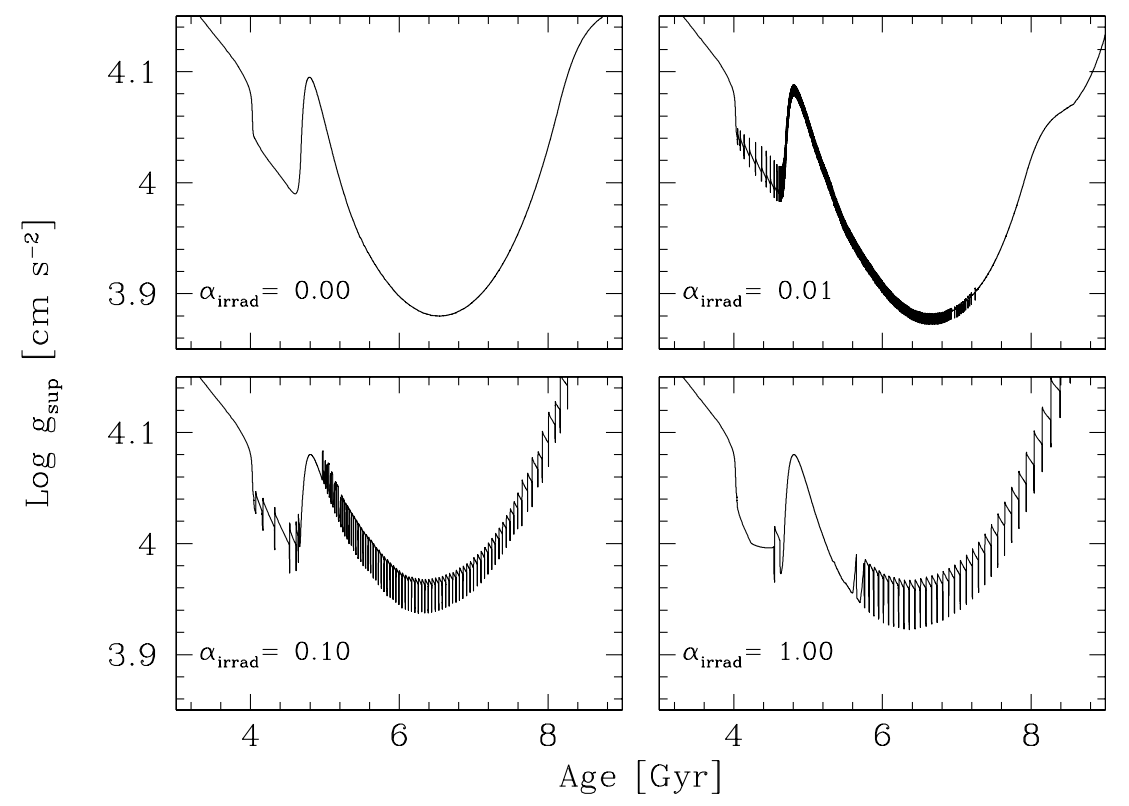

Figure 4. Surface gravity as a function of time for the systems included in Figure 2. These surface gravity values are similar to those expected for a main-sequence star, as suggested by Crawford et al. (2013) for the donor star of PSR J1723-2837 system.

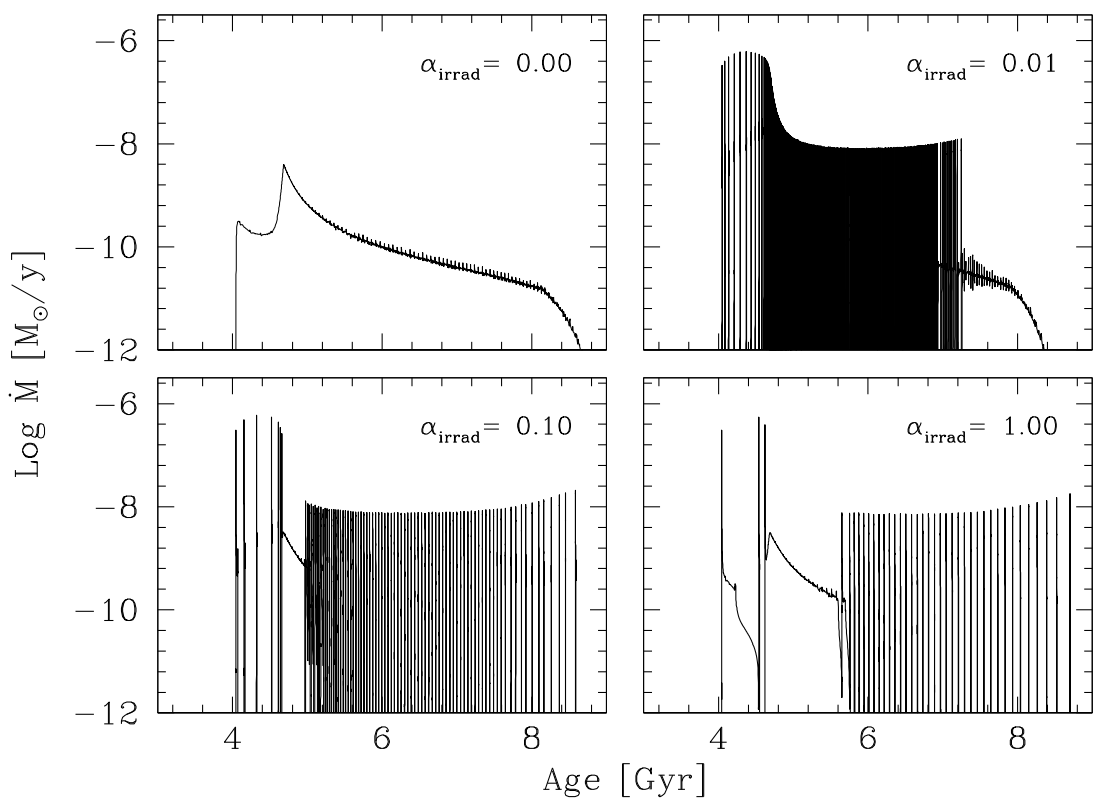

Figure 5. Mass-transfer rate for the systems included in Figure 2.

Thus, for different values of $\beta$, other consistent solutions should exist as well.

Some redback systems (PSR J1023+0038 and XSS J12270-4859) show evidence of surrounding material (Linares 2014) while others do not. In the framework of our models, they should correspond to the RLOF and quasi-RLOF states, respectively. Consequently, redbacks with surrounding material are revealed as pulsars, not due to the long-term evolution resulting from our calculations, but due to some physical processes (radio ejection and/or accretion disk instabilities) still to be included in the models. The very existence of redbacks with clean environments shows that irradiation feedback plays a key role in accounting for the evolution of these objects. If there were no clean redbacks, we could interpret that standard binary evolution coupled with radio ejection and/or accretion disk instabilities may be enough to account for observations. However, this does not seem to be the case.

The evolutionary status of redbacks has recently been addressed by Chen et al. (2013) employing models including evaporation. They state that the evolution leading to redbacks or black widows is determined by the efficiency of the irradiation process leading to mass loss via evaporation. They claim that redback systems do not evolve into black widow ones. This scenario is deeply different from the one described in BDVH, especially because Chen et al. (2013) do not find a stage similar to what we called the quasi-RLOF state, while, in the frame of our calculations, we interpret that all redbacks correspond to this state. Moreover, we found that some redbacks evolve into black widows (see BDVH for further details). 


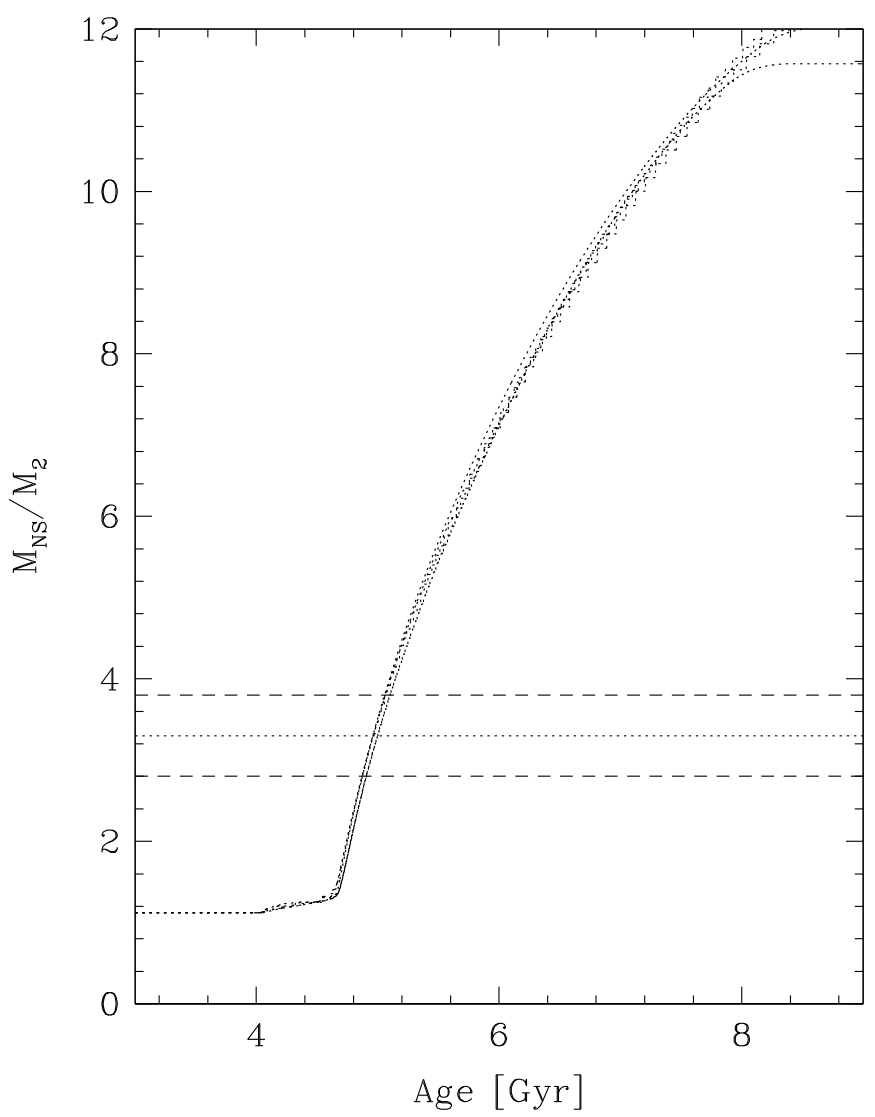

Figure 6. Ratio of the NS to donor mass as a function of time for the systems included in Figure 2. The mass ratio observed in the PSR J1723-2837 system is denoted with a dotted line, whereas its uncertainty is depicted with horizontal dashed lines. The models considered provide the correct mass ratio at an age of $\sim 4.9$ Gyr.

\section{CONCLUSIONS}

We have discussed the occurrence of the quasi-RLOF state originally discussed in BDVH and applied it to the well-studied redback system PSR J1723-2837 (Crawford et al. 2013). We found that low-irradiation models describe the properties of this binary system very well, suggesting that the quasi-RLOF state actually takes place along binary evolution. This also supports the relevance of irradiation feedback for these types of binary systems. It is likely that the phenomenology observed in the PSR J1723-2837 system, and in those quoted in the Introduction of this paper (Section 1), is representative of the whole class of redbacks.

Taken at face value, on human timescales, our models are able to account for the switch of a given system from LMXB to a binary radio pulsar, or in the opposite direction, only once. Detecting such a transition in the frame of our evolutionary models has an extremely low probability and would be very unlikely. However, in some systems (see Section 1), this switch has been observed from one state to the other and then back to the original. Therefore, a short $(\sim 1 \mathrm{yr})$ timescale is clearly present in these systems and does not stem from our theoretical framework. It seems natural to consider that the main ingredients to be included in the model to find such a short timescale for the switching behavior should be associated with the accretion disk surrounding the NS. It is known that accretion disks may undergo instabilities on short timescales (the disk instability model; see, e.g., Lasota 2001; Coriat et al. 2012) that may fall in the required range.

Recently, it has been possible to measure the orbital period change of SAX 1808.4-3658 (di Salvo et al. 2008; Burderi et al. 2009). The reported value of $\dot{P}_{\text {orb }}=(3.85 \pm 0.15) \times 10^{-12} \mathrm{ss}^{-1}$ is far larger than the value expected for conservative $(\beta=1)$ binary evolution. These authors interpret it as evidence for a strong mass loss from the system driven by radio ejection. While this may correspond to the case of SAX 1808.4-3658, it is worth

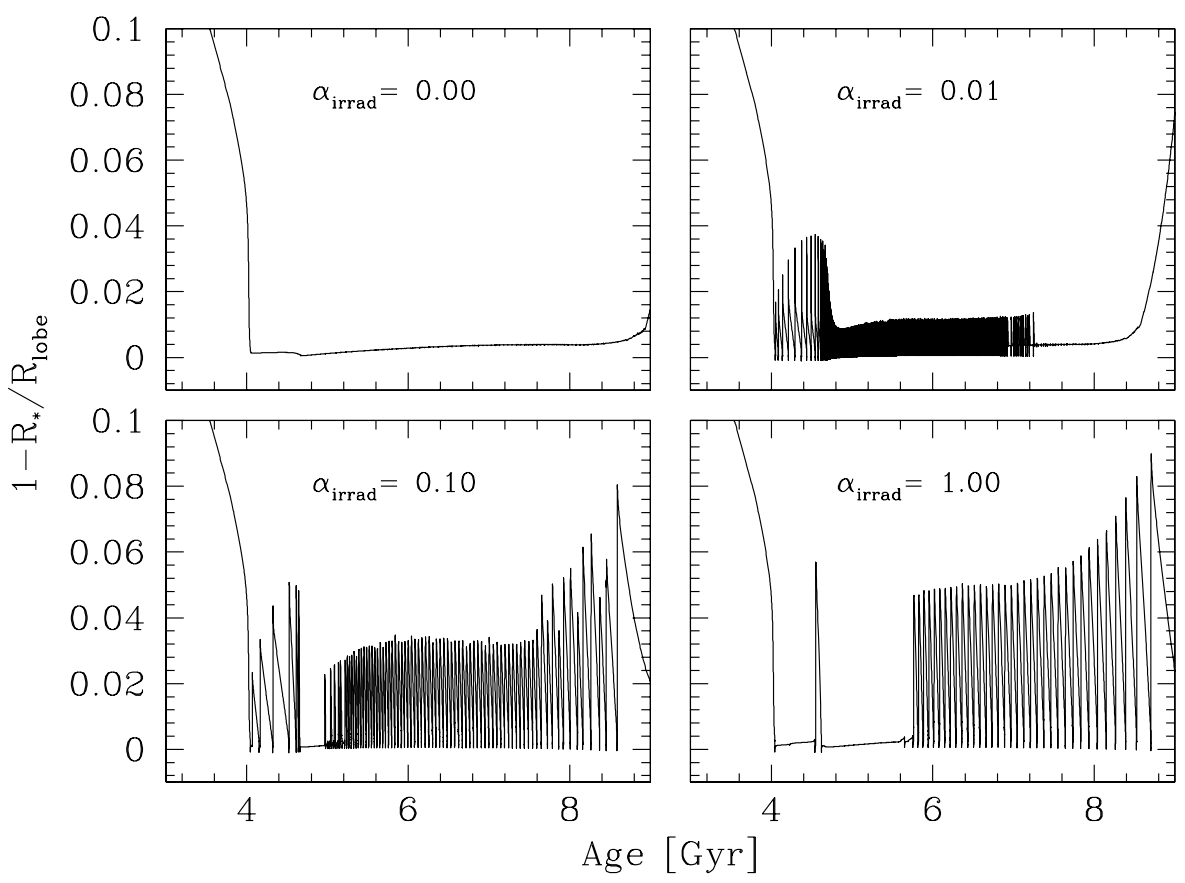

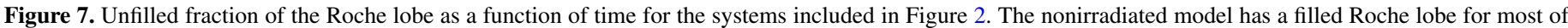

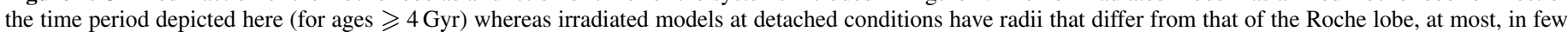
percents. The donor star is in the quasi-RLOF state. 
remarking that the radiation pressure of a radio pulsar does not necessarily destroy a surrounding accretion disk (Ekşi \& Alpar 2005).

Determining whether radio ejection and/or disk instability models are able to account for the observations described in Section 1 remains an open problem and deserves further investigation. Despite this, we consider that the framework for the occurrence of these complex phenomena is given by the long-term binary stellar evolution described in BDVH as well as in the present paper.

\section{REFERENCES}

Alpar, M. A., Cheng, A. F., Ruderman, M. A., \& Shaham, J. 1982, Natur, 300,728

Archibald, A. M., Stairs, I. H., Ransom, S. M., et al. 2009, Sci, 324, 1411

Bassa, C. G., Patruno, A., Hessels, J. W. T., et al. 2014, MNRAS, 441, 1825

Benvenuto, O. G., De Vito, M. A., \& Horvath, J. E. 2012, ApJL, 753, L33

Benvenuto, O. G., De Vito, M. A., \& Horvath, J. E. 2014, ApJL, 786, L7 (BDVH)

Benvenuto, O. G., \& De Vito, M. A. 2003, MNRAS, 342, 50

Benvenuto, O. G., \& De Vito, M. A. 2005, MNRAS, 362, 891

Benvenuto, O. G., Rohrmann, R. D., \& De Vito, M. A. 2006, MNRAS, 366,1520

Büning, A., \& Ritter, H. 2004, A\&A, 423, 281

Burderi, L., \& Di Salvo, T. 2013, MmSAI, 84, 117

Burderi, L., D’Antona, F., \& Burgay, M. 2002, ApJ, 574, 325

Burderi, L., Possenti, A., D’Antona, F., et al. 2001, ApJL, 560, L71

Burderi, L., Riggio, A., di Salvo, T., et al. 2009, A\&A, 496, L17

Chakrabarty, D., \& Morgan, E. H. 1998, Natur, 394, 346

Chen, H.-L., Chen, X., Tauris, T. M., \& Han, Z. 2013, ApJ, 775, 27
Crawford, F., Lyne, A. G., Stairs, I. H., et al. 2013, ApJ, 776, 20

Coriat, M., Fender, R. P., \& Dubus, G. 2012, MNRAS, 424, 1991

di Salvo, T., Burderi, L., Riggio, A., Papitto, A., \& Menna, M. T. 2008, MNRAS, 389, 1851

Ekşi, K. Y., \& Alpar, M. A. 2005, ApJ, 620, 390

Faulkner, A. J., Stairs, I. H., Kramer, M., et al. 2004, MNRAS, 355, 147

Fruchter, A. S., Stinebring, D. R., \& Taylor, J. H. 1988, Natur, 333, 237

Hameury, J.-M., \& Ritter, H. 1997, A\&AS, 123, 273

Lasota, J.-P. 2001, NewAR, 45, 449

Linares, M. 2014, ApJ, 795, 72

Papitto, A., Ferrigno, C., Bozzo, E., et al. 2013, Natur, 501, 517

Patruno, A., Archibald, A. M., Hessels, J. W. T., et al. 2014, ApJL, 781, L3

Phinney, E. S., Evans, C. R., Blandford, R. D., \& Kulkarni, S. R. 1988, Natur, 333,832

Podsiadlowski, P., Rappaport, S., \& Pfahl, E. D. 2002, ApJ, 565, 1107

Roberts, M. S. E. 2013a, IAU Symp. 291, Neutron Stars and Pulsars: Challenges and Opportunities after 80 Years, ed. J. van Leeuwen (Cambridge: Cambridge Univ. Press), 127

Roberts, M. S. E. 2013b, Talk given in the Workshop: Physical Applications of Millisecond Pulsars (Aspen, CO: Aspen Center for Physics), 2013 January 19-24, available at http://aspen13.phys.wvu.edu/aspen_talks/ Roberts_spiders.pdf

Romanova, M. M., Kulkarni, A. K., Long, M., \& Lovelace, R. V. E. 2008, in AIP Conf. Proc. 1068, A Decade of Accreting Millisecond X-ray Pulsars, ed. R. Wijnands et al. (Melville, NY: AIP), 87

Roy, J., Bhattacharyya, B., \& Ray, P. S. 2014, ATel, 5890, 1

Ruderman, M., Shaham, J., \& Tavani, M. 1989, ApJ, 336, 507

Stappers, B. W., Archibald, A. M., Hessels, J. W. T., et al. 2014, ApJ, 790, 39

Tauris, T. M., \& Savonije, G. J. 1999, A\&A, 350, 928

Thorstensen, J. R., \& Armstrong, E. 2005, AJ, 130, 759

Vaz, L. P. R., \& Nordlund, A. 1985, A\&A, 147, 281

Verbunt, F. 1993, ARA\&A, 31, 93

Webbink, R. F., Rappaport, S., \& Savonije, G. J. 1983, ApJ, 270, 678

Wijnands, R., \& van der Klis, M. 1998, Natur, 394, 344 